\title{
Adubação nitrogenada no desenvolvimento inicial de uvaia
}

\author{
Karen Andreon Viçosi ${ }^{1}$, Edvan Costa da Silva ${ }^{1}$, Franciely Magalhães Barroso ${ }^{1}$, João Paulo \\ Costa $^{1}$, Mariana Pina da Silva Berti ${ }^{1}$
}

${ }^{1}$ Universidade Estadual de Goiás, Campus de Ipameri, Ipameri, Goiás, Brasil. E-mail: karen_vicosi@hotmail.com, edvan_costa@outlook.com,fran_magb@hotmail.com,joaopaulo_mc@hotmail.com,mariana.berti@ueg.com

Recebido: 18/04/2018; Aceito: 04/01/2019.

\section{RESUMO}

A uvaia é uma espécie arbórea nativa utilizada tanto na indústria, quanto no paisagismo e reflorestamento, porém, pouco estudada em relação ao seu manejo, principalmente quando se refere à adubação. Objetivou-se avaliar o efeito de diferentes doses de nitrogênio no transplantio de mudas de Eugenia pyriformis. O experimento foi desenvolvido em casa de vegetação na Universidade Estadual de Goiás, Câmpus Ipameri-GO. Foi utilizado o delineamento inteiramente casualizado, com seis doses de nitrogênio na ocasião do transplantio $(0,50,100,150$, 200 e $250 \mathrm{~kg} \mathrm{ha}^{-1}$ ), na forma de ureia, com cinco repetições por tratamento. As plantas foram avaliadas aos $30 \mathrm{e}$ 60 dias após o transplantio, obtendo as variáveis altura; diâmetro do caule; número de galhos; massa seca da parte aérea e relação altura/diâmetro. A utilização de diferentes doses de nitrogênio não influenciou no crescimento inicial da uvaia para altura, diâmetro, número de galhos e massa seca. Quando comparado às variáveis analisadas aos 30 e 60 dias, observa-se que houve incremento do diâmetro e aumento na relação altura/diâmetro, independente da dose de nitrogênio. A adubação nitrogenada no transplantio de mudas não interfere no crescimento inicial da espécie, ocorrendo uma estabilização dos valores de altura e incremento do diâmetro da planta aos 60 dias de transplantio.

Palavras-chave: Eugenia pyriformis, ureia, espécie nativa, transplantio.

\section{Nitrogen fertilization in the initial development of uvaia}

\begin{abstract}
The uvaia is a native tree concept for industry, landscaping and reforestation, little studied in relation to its management, most related to fertilization. The objective of this work was to evaluate the effect of different doses of nitrogen on the transplanting of Eugenia pyriformis seedlings. The experiment was conducted in a greenhouse at the State University of Goiás, Campus Ipameri-GO. A completely randomized design, with 6 nitrogen doses at the time of transplanting $\left(0,50,100,150,200\right.$ and $\left.250 \mathrm{~kg} \mathrm{ha}^{-1}\right)$, in urea form with five replications per treatment was used. The plants were evaluated at 30 days and 60 days after transplantation, obtaining the height; stem diameter; number of branches; dry mass and relation height/diameter. The use of different doses of nitrogen did not influence the initial growth of uvaia for height, diameter, number of branches and dry mass. When comparing the variables analyzed at 30 and 60 days, there was an increase in diameter and increase in the relation height/diameter, regardless of the nitrogen dose. Nitrogen fertilization in the transplanting of seedlings does not interfere with the initial growth of the species, resulting in a stabilization of height and increment of plant diameter at 60 days of transplanting.
\end{abstract}

Keywords: Eugenia pyriformis, urea, native species, transplanting. 


\section{Introdução}

A uvaia (Eugenia pyriformis) é uma árvore pertencente à família Myrtaceae, nativa dos biomas do Cerrado e Mata Atlântica, de hábito arbóreo e mede de 5 a $15 \mathrm{~m}$ de altura (Scalon et al., 2012). A espécie pode ser utilizada para recomposição de florestas nativas, em projetos paisagísticos e como árvore frutífera, devido aos seus frutos de coloração amarela, com sabor adocicado e acidulado, com ótimas propriedades nutricionais e físico-químicas, utilizados pela indústria na forma de produtos processados como geleias e sucos (Souza et al., 2009; Sganzerla et al., 2018).

Com a atual redução da porcentagem de matas nativas devido ao desmatamento para abertura de áreas para empreendimentos agropecuários e econômicos, há pouco conhecimento na literatura sobre as frutíferas nativas da região do cerrado, ocasionando falta de informações em relação aos benefícios à saúde, ao meio ambiente e à geração de renda (Zillo et al., 2013). Contudo, com o aumento da demanda por mudas nativas devido à exigência legislativa de propriedades rurais para a adequação de reserva legal e áreas de preservação permanente, faz-se imprescindível expandir os estudos silviculturais destas espécies para promover seu melhor desenvolvimento e garantia de sobrevivência em campo (Dalanhol et al., 2012).

Dentre os diversos elementos do manejo de plantio de espécies nativas, a adubação de plantio, sem dúvida, é um fator importantíssimo para o estabelecimento e desenvolvimento da cultura. Segundo Rosa et al. (2014), a adubação das árvores faz-se necessária, principalmente em solos do Cerrado, que é composto de solos ácidos, com pouca matéria orgânica do solo e, em muitos casos, não fornece as quantidades totais de nutrientes que as plantas necessitam para se desenvolver e ter um crescimento inicial satisfatório.

A escassez de estudos envolvendo a absorção de nutrientes e requerimentos nutricionais de espécies florestais nativas é um dos maiores entraves para o seu uso em plantios comerciais ou na recuperação de áreas degradadas (Bernadino et al., 2005). De acordo com Tucci et al. (2011), há uma necessidade contínua de estudos para conciliar o manejo adequado da nutrição mineral no crescimento inicial de árvores nativas, de modo a reduzir o custo de implantação das espécies florestais e racionalizar o uso de fertilizantes.

Diante disto, o objetivo do presente trabalho foi avaliar o efeito de diferentes doses de nitrogênio no transplantio de mudas de Eugenia pyriformis.

\section{Material e Métodos}

O experimento foi desenvolvido em casa de vegetação na Universidade Estadual de Goiás, Câmpus Ipameri, localizada no município de Ipameri-GO $\left(17^{\circ}\right.$ 43' S, $48^{\circ} 22^{\prime}$ 'W e altitude de $800 \mathrm{~m}$, durante o período de novembro de 2017 a fevereiro de 2018. O clima, segundo a classificação de Köppen, é definido como Tropical Úmido (AW), constando temperaturas elevadas com chuvas no verão e seca no inverno.

O substrato utilizado foi o Latossolo Vermelho Amarelo distrófico, coletado da Fazenda Experimental da UEG na camada subsuperficial. Após a coleta, as amostras de solo foram secas à sombra sobre lona de plástico, por um período de doze dias, em seguida peneiradas (malha de $4 \mathrm{~mm}$ ) e homogeneizadas para realização da análise química solo, descrita na Tabela 1 .

Foi utilizado o delineamento inteiramente casualizado, com seis tratamentos correspondentes a diferentes doses de nitrogênio $(0,50,100,150,200$ e $250 \mathrm{~kg} \mathrm{ha}^{-1}$ ) na forma de ureia, com cinco repetições.

As mudas de uvaia foram transplantadas em vasos preenchidos com 9 litros de solo corrigido com calcário, a fim de elevar a saturação de bases a $60 \%$, e posteriormente adubados no plantio com $200 \mathrm{~kg} \mathrm{ha}^{-1} \mathrm{de}$ $\mathrm{P}_{2} \mathrm{O}_{5}$ e $200 \mathrm{~kg} \mathrm{ha}^{-1}$ de $\mathrm{K}_{2} \mathrm{O}$, com irrigação diária.

Aos 30 dias após o transplantio, as plantas foram analisadas para obtenção das variáveis: altura média da parte aérea $(\mathrm{cm})$, através de régua graduada; diâmetro do caule $(\mathrm{mm})$, com paquímetro digital; número de galhos, obtido por contagem manual. Aos 60 dias, além dessas variáveis, foi também analisada massa seca média da parte aérea $(\mathrm{g})$, em estufa até peso constante a $65-70{ }^{\circ} \mathrm{C}$. Com os dados obtidos, foi obtida a relação altura/diâmetro do coleto (R/DC), resultado da divisão da altura pelo diâmetro.

Tabela 1 - Propriedades químicas do solo determinadas antes da instalação do experimento, coletadas na área experimental da Universidade Estadual de Goiás, Câmpus Ipameri.

\begin{tabular}{cccccc}
\hline Determinação & Unidade & Valor & Determinação & Unidade & Valor \\
\hline $\mathrm{pH}$ & $\mathrm{CaCl}_{2}$ & 4,4 & $\mathrm{CTC}$ & $\mathrm{mmolc}_{\mathrm{cm}}^{-3}$ & 59 \\
$\mathrm{H}+\mathrm{Al}$ & $\mathrm{mmol}_{\mathrm{c} \mathrm{dm}} \mathrm{dm}^{-3}$ & 47 & $\mathrm{~V}$ & $\%$ & 21 \\
$\mathrm{Ca}$ & $\mathrm{mmolc} \mathrm{dm}^{-3}$ & 7 & $\mathrm{Cu}$ & $\mathrm{mg} \mathrm{dm}^{-3}$ & 1,2 \\
$\mathrm{Mg}$ & $\mathrm{mmol}_{\mathrm{c} \mathrm{dm}} \mathrm{dm}^{-3}$ & 3 & $\mathrm{Fe}$ & $\mathrm{mg} \mathrm{dm}^{-3}$ & 66 \\
$\mathrm{P}$ (resina) & $\mathrm{mg} \mathrm{dm}^{-3}$ & 5 & $\mathrm{Mn}$ & $\mathrm{mg} \mathrm{dm}^{-3}$ & 6,0 \\
$\mathrm{~K}$ & $\mathrm{mmol}_{\mathrm{c} \mathrm{dm}}^{-3}$ & 2,4 & $\mathrm{Zn}$ & $\mathrm{mg} \mathrm{dm}^{-3}$ & 0,6 \\
M.O. & $\mathrm{g} \mathrm{dm}^{-3}$ & 33 & $\mathrm{~B}$ & $\mathrm{mg} \mathrm{dm}^{-3}$ & 0,12 \\
\hline
\end{tabular}


Os dados obtidos dos 30 e 60 dias foram comparados entre si, dentro de cada dose de nitrogênio, através do Teste $\mathrm{T}$ a $5 \%$ de probabilidade. Os resultados da análise aos 60 dias foram submetidos à análise de regressão. Todas as análises foram realizadas através do software R (Ferreira et al., 2013).

\section{Resultados e Discussão}

A utilização de diferentes doses de nitrogênio não influenciou no crescimento inicial da uvaia, obtendo-se resultado não significativo para todas as variáveis analisadas, conforme Figuras 1 e 2 .

Resultado semelhante foi encontrado por Souza et al. (2009), que também encontraram efeito não significativo para altura, comprimento de raiz, número de folhas e massa seca de uvaia submetidos a diferentes doses de nitrogênio ainda na fase de formação de muda.

Quando comparado a outras espécies da família Myrtaceae, resultados divergentes são encontrados na literatura. Jesus et al. (2016) encontraram aumento da altura, diâmetro e número de folhas com a utilização da adubação nitrogenada, corroborando com o resultado encontrado por Dias et al. (2012), que concluíram que a adubação nitrogenada na cultura da goiabeira é um fator importante no crescimento inicial da planta.

Para o camu-camu, doses acima de 0,16 $\mathrm{gL}^{-1}$ prejudicam o desenvolvimento inicial e formação das mudas dessa cultura (Santos et al., 2012), enquanto que para a pintangueira (E. uniflora) recomenda-se iniciar a adubação após o segundo mês, quando começou um incremento tanto em altura como em diâmetro do colo em resposta à adubação nitrogenada (Dalanhol et al., 2012).

Observa-se que espécies cultivadas comercialmente, que sofreram processo de melhoramento e seleção de cultivares, respondem à adubação nitrogenada, enquanto que para espécies nativas, ainda pouco estudadas, a adubação nitrogenada não se constitui um fator fundamental na fase de mudas e desenvolvimento inicial da planta.
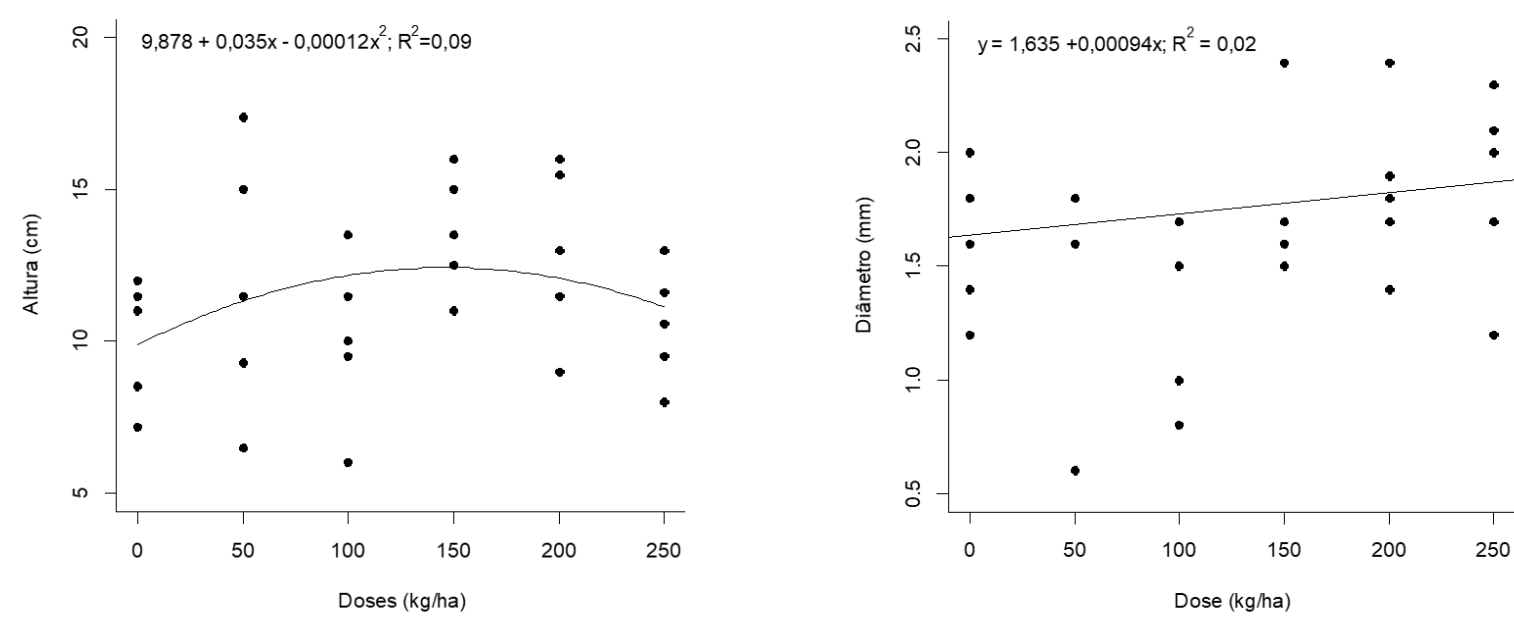

Figura 1 - Altura (cm) e diâmetro do coleto $(\mathrm{mm})$ em função de crescentes doses de nitrogênio em plantas de uvaia.
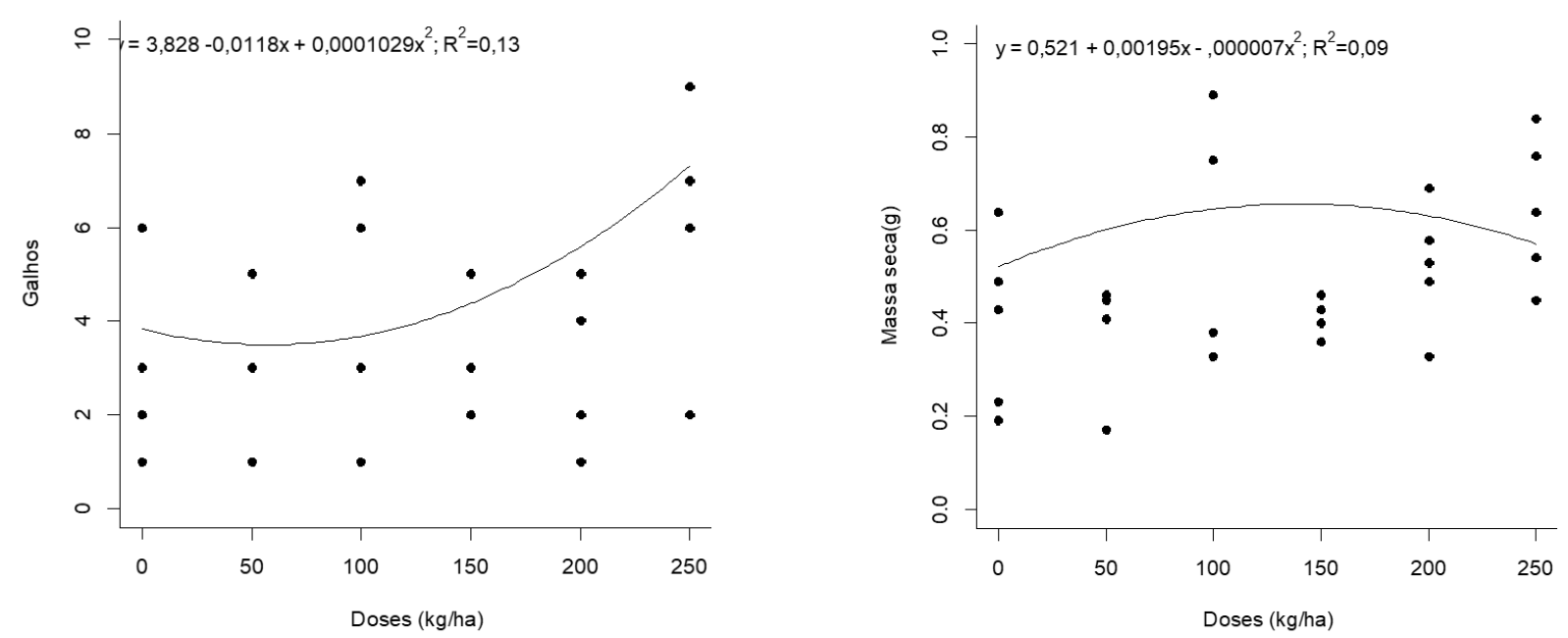

Figura 2 - Número de galhos e massa seca da parte aérea (g) em função de crescentes doses de nitrogênio em plantas de uvaia. 
Quando comparado às variáveis analisadas aos $30 \mathrm{e}$ 60 dias (Tabela 1), observa-se que para altura e número de galhos não houve diferença significativa entre as duas análises, sob diferentes doses de nitrogênio. Entretanto, houve incremento significativo do diâmetro e aumento na relação altura/diâmetro para todas as doses de 0, 150, 200 e $250 \mathrm{~kg} \mathrm{ha}^{-1}$. Pode-se inferir, então, que para a cultura da uvaia o crescimento inicial se resume ao incremento do diâmetro, independente ou não da utilização de adubação nitrogenada. De acordo com Gomes et al. (2013), o diâmetro é uma característica fundamental na produção de mudas e para a avaliação da capacidade de sobrevivência destas em campo, relacionando o maior incremento em diâmetro com maior capacidade de formação e de crescimento de novas raízes.
A relação altura/coleto demonstra o equilíbrio de crescimento das mudas, também denominado de quociente de robustez, sendo este um dos parâmetros mais utilizados para avaliar a qualidade de mudas florestais, por refletir o acúmulo de reservas, assegurar maior resistência ao déficit hídrico e melhor fixação no solo (Abreu et al., 2015).

Caldeira et al. (2008) recomendam que esta relação se mantenha abaixo de 10 , sendo que valores acima deste implicam em estiolamento da muda e menor índice de sobrevivência no campo. Sendo assim, observou-se que aos 30 dias (Tabela 2), para todas as doses estudadas, as mudas de uvaia estavam estioladas, e, aos 60 dias, a relação se tornou ideal devido à estabilização do crescimento em altura e incremento do diâmetro do caule.

Tabela 2 - Altura $(\mathrm{cm})$, diâmetro do coleto $(\mathrm{mm})$, relação altura/diâmetro (H/DC) e número de galhos em plantas de uvaia submetidas a diferentes doses de nitrogênio, aos 30 dias (30 D) e 60 dias (60 D).

\begin{tabular}{|c|c|c|c|c|c|c|c|c|c|c|c|}
\hline \multicolumn{12}{|c|}{ Altura (cm) } \\
\hline \multicolumn{2}{|c|}{$0 \mathrm{~kg} \mathrm{ha}^{-1}$} & \multicolumn{2}{|c|}{$50 \mathrm{~kg} \mathrm{ha}^{-1}$} & \multicolumn{2}{|c|}{$100 \mathrm{~kg} \mathrm{ha}^{-1}$} & \multicolumn{2}{|c|}{$150 \mathrm{~kg} \mathrm{ha}^{-1}$} & \multicolumn{2}{|c|}{$200 \mathrm{~kg} \mathrm{ha}^{-1}$} & \multicolumn{2}{|c|}{$250 \mathrm{~kg} \mathrm{ha}^{-1}$} \\
\hline $30 \mathrm{D}$ & $60 \mathrm{D}$ & $30 \mathrm{D}$ & $60 \mathrm{D}$ & $30 \mathrm{D}$ & $60 \mathrm{D}$ & $30 \mathrm{D}$ & $60 \mathrm{D}$ & $30 \mathrm{D}$ & $60 \mathrm{D}$ & $30 \mathrm{D}$ & $60 \mathrm{D}$ \\
\hline $10,0 \mathrm{a}$ & $10,3 \mathrm{a}$ & $11,9 \mathrm{a}$ & $12,0 \mathrm{a}$ & $9,3 \mathrm{a}$ & $10,1 \mathrm{a}$ & $12,8 \mathrm{a}$ & $13,1 \mathrm{a}$ & $12,3 \mathrm{a}$ & $13,0 \mathrm{a}$ & $9,0 \mathrm{a}$ & $10,5 \mathrm{a}$ \\
\hline \multicolumn{12}{|c|}{ Diâmetro (mm) } \\
\hline \multicolumn{2}{|c|}{$0 \mathrm{~kg} \mathrm{ha}^{-1}$} & \multicolumn{2}{|c|}{$50 \mathrm{~kg} \mathrm{ha}^{-1}$} & \multicolumn{2}{|c|}{$100 \mathrm{~kg} \mathrm{ha}^{-1}$} & \multicolumn{2}{|c|}{$150 \mathrm{~kg} \mathrm{ha}^{-1}$} & \multicolumn{2}{|c|}{$200 \mathrm{~kg} \mathrm{ha}^{-1}$} & \multicolumn{2}{|c|}{$250 \mathrm{~kg} \mathrm{ha}^{-1}$} \\
\hline $30 \mathrm{D}$ & $60 \mathrm{D}$ & $30 \mathrm{D}$ & $60 \mathrm{D}$ & $30 \mathrm{D}$ & $60 \mathrm{D}$ & $30 \mathrm{D}$ & $60 \mathrm{D}$ & $30 \mathrm{D}$ & $60 \mathrm{D}$ & $30 \mathrm{D}$ & $60 \mathrm{D}$ \\
\hline $0,7 \mathrm{~b}$ & $1,6 \mathrm{a}$ & $1,0 \mathrm{a}$ & $1,8 \mathrm{a}$ & $0,8 \mathrm{a}$ & $1,7 \mathrm{a}$ & $0,8 \mathrm{~b}$ & $1,8 \mathrm{a}$ & $0,8 b$ & $1,8 \mathrm{a}$ & $0,7 \mathrm{~b}$ & $1,8 \mathrm{a}$ \\
\hline \multicolumn{12}{|c|}{ Relação H/DC (cm) } \\
\hline \multicolumn{2}{|c|}{$0 \mathrm{~kg} \mathrm{ha}^{-1}$} & \multicolumn{2}{|c|}{$50 \mathrm{~kg} \mathrm{ha}^{-1}$} & \multicolumn{2}{|c|}{$100 \mathrm{~kg} \mathrm{ha}^{-1}$} & \multicolumn{2}{|c|}{$150 \mathrm{~kg} \mathrm{ha}^{-1}$} & \multicolumn{2}{|c|}{$200 \mathrm{~kg} \mathrm{ha}^{-1}$} & \multicolumn{2}{|c|}{$250 \mathrm{~kg} \mathrm{ha}^{-1}$} \\
\hline $30 \mathrm{D}$ & $60 \mathrm{D}$ & $30 \mathrm{D}$ & $60 \mathrm{D}$ & $30 \mathrm{D}$ & $60 \mathrm{D}$ & $30 \mathrm{D}$ & $60 \mathrm{D}$ & $30 \mathrm{D}$ & $60 \mathrm{D}$ & $30 \mathrm{D}$ & $60 \mathrm{D}$ \\
\hline $14,6 \mathrm{a}$ & $6,4 \mathrm{~b}$ & $12,3 \mathrm{a}$ & $7,5 \mathrm{a}$ & $13,4 \mathrm{a}$ & $7,3 \mathrm{a}$ & $12,2 \mathrm{a}$ & $7,7 \mathrm{~b}$ & $15,9 \mathrm{a}$ & $7,3 \mathrm{~b}$ & $13,6 \mathrm{a}$ & $6,2 \mathrm{~b}$ \\
\hline \multicolumn{12}{|c|}{ Galhos (cm) } \\
\hline \multicolumn{2}{|c|}{$0 \mathrm{~kg} \mathrm{ha}^{-1}$} & \multicolumn{2}{|c|}{$50 \mathrm{~kg} \mathrm{ha}^{-1}$} & \multicolumn{2}{|c|}{$100 \mathrm{~kg} \mathrm{ha}^{-1}$} & \multicolumn{2}{|c|}{$150 \mathrm{~kg} \mathrm{ha}^{-1}$} & \multicolumn{2}{|c|}{$200 \mathrm{~kg} \mathrm{ha}^{-1}$} & \multicolumn{2}{|c|}{$250 \mathrm{~kg} \mathrm{ha}^{-1}$} \\
\hline $30 \mathrm{D}$ & $60 \mathrm{D}$ & $30 \mathrm{D}$ & $60 \mathrm{D}$ & $30 \mathrm{D}$ & $60 \mathrm{D}$ & $30 \mathrm{D}$ & $60 \mathrm{D}$ & $30 \mathrm{D}$ & $60 \mathrm{D}$ & $30 \mathrm{D}$ & $60 \mathrm{D}$ \\
\hline $2,2 \mathrm{a}$ & $2,8 \mathrm{a}$ & $4,6 \mathrm{a}$ & $5,2 \mathrm{a}$ & $4,0 \mathrm{a}$ & $4,8 \mathrm{a}$ & $3,4 \mathrm{a}$ & $5,0 \mathrm{a}$ & $4,0 \mathrm{a}$ & $4,8 \mathrm{a}$ & $5,0 \mathrm{a}$ & $8,0 \mathrm{a}$ \\
\hline
\end{tabular}

Médias seguidas pela mesma letra em uma mesma dose não diferem entre si pelo Teste $\mathrm{T}$ a $5 \%$ de probabilidade.

\section{Conclusões}

A adubação nitrogenada no transplantio de mudas não interfere no crescimento inicial da espécie. Há uma estabilização do crescimento da altura e incremento do diâmetro da planta aos 60 dias de transplantio.

\section{Referências Bibliográficas}

Abreu, A.J.M., Leles, P.S.S., Melo, L.A., Ferreira, D.H.A.A., Monteiro, F.A.S., 2015. Produção de mudas e crescimento inicial em campo de Enterolobium contortisiliquum produzidas em diferentes recipientes. Floresta, 45, 141-150.

Bernadino, D.C.S., Paiva, H.N., Neves, J.C.L., Gomes, J.M., Marques, V.B., 2005. Crescimento e qualidade de mudas de Anadenanthera macrocarpa (Benth.) Brenan em resposta à saturação por bases do substrato. Árvore, 29, 863-870.
Caldeira, M.V.W., Rosa, G.N., Fenilli, T.A.B., Harbs, R.M.P., 2008. Composto orgânico na produção de mudas de aroeiravermelha. Scientia Agraria, 9, 27-33.

Dalanhol, S.J., Kratz, D., Nogueira, A.C., Gaiad, S., 2012. Efeito da adubação no crescimento de mudas de Eugenia uniflora L. In: FERTBIO, 2012, Maceió-AL. Anais... ViçosaMG: SBCS, 2012. 3 p.

Dias, M.J.T., Souza, H.A., Natale, W., Modesto, V.C., Rozante, D.E, 2012. Adubação com nitrogênio e potássio em mudas de goiabeira em viveiro comercial. Semina: Ciências Agrárias, 33, 2837-2848.

Ferreira, E.B., Cavalcanti, P.P., Nogueira, D.A., 2013. ExpDes: Experimental Designs pacakge. $\mathrm{R}$ package version 1.1.2.

Gomes, D.R., Caldeira, M.V.W., Delarmelina, W.M., Gonçalves, E.O., Trazzi, P.A., 2013. Lodo de esgoto como 
substrato para produção de mudas de Tectona grandis L. Cerne, 19, 123-131.

Jesus, M.S., Fernandes, L.A., Fonseca, F.S.A., Rocha, S.M.G., Martins, E.R., 2016. Crescimento e composição química do óleo essencial de mudas de Eucalyptus globulus (Labill) adubadas com nitrogênio e potássio. Caderno de Ciências Agrárias, 8, 01-08.

Rosa, R., Sano, E.E., Rosendo, J.S., 2014. Estoque de carbono em solos sob pastagens cultivadas na bacia hidrográfica do rio Paranaíba. Sociedade e Natureza, 26, 333-351.

Scalon, S.P.Q, Neves, E.M.S., Maseto, T.E., Pereira, Z.V., 2012. Sensibilidade à dessecação e ao armazenamento em sementes de Eugenia pyriformis Cambess. (uvaia). Revista Brasileira de Fruticultura, 34, 269-276.

Santos, V.A., Chagas, E.A., Vilena, J.O., Lozano, R.M.B., Ribeiro, I.G., Albuquerque, R.C.S., 2012. Adubação nitrogenada na formação de mudas de camu-camu. In: $1^{\circ}$ Simpósio Internacional de Botânica Aplicada e $1^{\circ}$ Simpósio
Nacional de Frutíferas do Norte e Nordeste, 2012, Lavras-MG. Anais... Lavras-MG: CPAF, 2012. p. 10.

Sganzerla, W.G., Beling, P.C., Ferrareze, J.P., Komatsu, R.A., Nunes, M.R., Veeck, A.P.L., 2018. Nutritional, physicochemical and antimicrobial properties of uvaia pulp (Eugenia pyriformis Cambess). Communications in Plant Sciences, 8, 1-7.

Souza, H.A., Gurgel, R.L.S., Teixeira, G.A., Cavallari, L.L., Rodrigues, H.C.A., Mendonça, V., 2009. Adubação nitrogenada e fosfatada no desenvolvimento de mudas de uvaia. Bioscience Journal, 25, 99-103.

Tucci, C.A.F., Santos, J.Z.L., Silva Júnior, C.H., Souza, P.A., Batista, I.M.P., Venturin, N., 2011. Desenvolvimento de mudas de Swietenia macrophylla em resposta a nitrogênio, fósforo e potássio. Floresta, 41, 471-490.

Zillo, R.R., Silva, P.P.M., Zanatta, S., Carmo, L.F., Spoto, M.H.F. Qualidade físico-química da fruta in natura e da polpa de uvaia congelada. Revista Brasileira de Produtos Agroindustriais, 15, 293-298. 